$56^{\text {ème }}$ Congrès de la SFMBCB, 03018 (2011)

DOI: $10.1051 / \mathrm{sfmbcb} / 20115603018$

(C) Owned by the authors, published by EDP Sciences, 2011

\title{
Suivi odontologique des patients diabétiques en milieu hospitalier
}

\author{
Triay JA, Joly JP, Delarbre B \\ Service d'Odontologie et de Prothèse maxillo-faciale, CHU, Nîmes, France \\ jatriay@hotmail.com
}

Les diabétiques sont des patients à risque qui doivent être suivis tout particulièrement et conjointement par le médecin et le chirurgien-dentiste (bulletin de l'Académie Nationale de Chirurgie Dentaire 2007). Le Service d’Odontologie et le Service des Maladies métaboliques et endocriniennes (MME) du CHU de Nîmes ont constitué en 1998 une équipe chargée de la prévention et du dépistage bucco-dentaires des patients diabétiques, dès la consultation initiale et lors des séances de contrôle et de suivi du diabète.

L’objectif de cette analyse statistique est d'évaluer l'efficacité de cette action de médecine transversale en termes de soins odontologiques hospitaliers, mais aussi de réorientation vers l'odontologie de ville. Une grille d'évaluation permet d'enregistrer des observations cliniques simples afin d'établir le status buccal de chaque patient hospitalisé et d'identifier les patients à risques. Sont ainsi précisées les indications de soins (chirurgie buccale, bilan complémentaire, parodontie, prothèse, pathologie de l'articulation temporo-mandibulaire). On note enfin l'orientation conseillée : les patients dépistés en MME sont soit adressés à leur chirurgien dentiste traitant, soit confiés au Service d'Odontologie.

Résultats : Sur la période 2006-2009, 1147 patients ont été dépistés et 22,5\% ont été orientés pour une prise en charge bucco-dentaire ou un bilan complémentaire. Parmi eux, 6,7\% ont été confiés, avec un courrier, à leur praticien traitant et, pour 13,6\%, il a été proposé une consultation ou une prise en charge dans le Service d'Odontologie. Cette dernière était motivée dans 40,6\% des cas par une indication de chirurgie buccale ou un bilan buccodentaire approfondi (38\%).

Chez ces patients qui devraient percevoir l'hygiène et le bon état buccal comme une priorité, les besoins en soins vont bien au-delà des affections classiquement associées au diabète. Le dépistage des problèmes bucco-dentaires au sein du service de MME est l'occasion à la fois de traiter, d'informer le patient et de le remettre dans un circuit de soins et de contrôles réguliers qui doivent se faire hors de toute hospitalisation.

La capacité des Services hospitaliers d'Odontologie à devenir des pôles de référence, de coordination et de ressources et l'activation du lien entre la ville et l'hôpital constituent un enjeu formidable pour la médecine bucco-dentaire. 